\title{
Brain white matter fiber estimation and tractography using Q-ball imaging and Bayesian MODEL
}

\author{
Meng Lu* \\ College of Information Science and Engineering, Northeastern University, China
}

\begin{abstract}
Diffusion tensor imaging allows for the non-invasive in vivo mapping of the brain tractography. However, fiber bundles have complex structures such as fiber crossings, fiber branchings and fibers with large curvatures that tensor imaging (DTI) cannot accurately handle. This study presents a novel brain white matter tractography method using Q-ball imaging as the data source instead of DTI, because QBI can provide accurate information about multiple fiber crossings and branchings in a single voxel using an orientation distribution function (ODF). The presented method also uses graph theory to construct the Bayesian model-based graph, so that the fiber tracking between two voxels can be represented as the shortest path in a graph. Our experiment showed that our new method can accurately handle brain white matter fiber crossings and branchings, and reconstruct brain tractograhpy both in phantom data and real brain data.
\end{abstract}

Keywords: Brain white matter, graph theory, QBI; tractography, Bayesian model

\section{Introduction}

Diffusion tensor imaging (DTI) is a magnetic resonance imaging method that can measure the anisotropic diffusion of water molecules of in vivo biological tissue such as white matter(WM) in the brain $[1,2]$. These indirect observations of white matter geometry allow nerve fiber reconstruction through the use of tractography algorithms [3]. The reconstruction of white matter fibers in the human brain can be used to investigate connectivity in mental and neurological disorders. Another application of white matter tractography is surgical planning [4].

Basser, et al. proposed the first tractography method [5]. White matter fiber paths were estimated by tracing the direction of maximal water diffusion using diffusion weighted magnetic resonance imaging (MRI), with stop criteria of low Fractional Anisotropy (FA) values or maximum curvature. Basser's method is the representative work of local and deterministic tractography algorithms, which can be influenced by the accumulation of errors and noise in the diffusion data. To overcome these problems, the global optimal framework for brain tractography was proposed [6-10]. Global parameters were optimized using the diffusion data and some constraints to get global fiber bundles [3]. However, the global optimal framework for brain tractoraphy is computationally expensive because an optimized

* Address for correspondence: Meng Lu, College of Information Science and Engineering, Northeastern University, China. Tel.: 18602422117; E-mail: menglu1982@gmail.com. 
estimation must be made for the entirety of the fiber bundles. Another way to overcome the issue of noise and partial volume effect in the diffusion data is to use a probabilistic framework, which characterizes the uncertainty in fiber path estimation with a set of possible propagation directions for a given voxel [3]. Probabilistic methods can generate multiple fiber trajectories with different probabilities and a probability map from the seed voxel to the other voxels in the brain [11-13].

However, even with these techniques, complex fiber architectures such as fiber crossing, kissing, and branching cannot be accurately characterized and segmented. To deal with this issue, Iturria-Medina, et al. [14] used graph theory to build a framework for brain white matter fiber tracking using DTI. All of the brain diffusion data were transformed into a weighted graph, and the fiber tracking was transformed to locate the most probable path in the graph. N.S. Stamatios, et al. used QBI in the graph theory instead of DTI and built a more complex graph to characterize fiber crossing, kiss, and branching using orientation distribution functions (ODF). Not just brain white matter tractography, graph theory was also used on cardiac tractography [15]. Building off these previous studies, our study presents a novel brain tractography algorithm using High Angular Resolution Diffusion Imaging (HARDI) and graph theory. HARDI can provide more information about ODF than diffusion tensor imaging, and Q-Ball Imaging (QBI) is used to model HARDI. We used the graph framework to characterize the connectivity between voxels in the brain white matter. Our method is an improvement from the previous studies because each voxel is transformed into a node on a weighted graph, and the weight of the edges are calculated based on Bayesian model and white matter ODF.

\section{Method}

\section{1. $Q B I$ and $O D F$}

Complex brain white matter fiber architectures (such as fiber crossing, fiber kissing, fiber branching, etc.) are widespread throughout the human brain [11], and traditional tensor models cannot adequately handle their fiber bundle geometry [3]. Therefore, the HARDI framework was proposed to deal with these issues. The HARDI framework has high angular resolution of the acquisition protocol, and the increased diffusion sensitization is able to capture subtle diffusion displacements of water molecules in order to obtain more information about diffusion directions in in a single voxel.

Based on HARDI, QBI was first proposed by Tuch [16], and is obtained by a projection on the Q-space using a Funk-Radon transform. In contrast to the single-peaked Gaussian ODF model used in diffusion tensor imaging [5], Q-ball imaging can reconstruct multi-peaked ODFs in cases of intra-voxel fiber crossings, kissing or branching [17], which provide a feasible manner to characterize complex brain white matter fiber architectures. The presented method used spherical deconvolution [18] and normalization [19] to improve the accuracy and robustness of the Q-ball model. Further details about QBI and ODF can be found in [3] and [20].

\subsection{Brain graph construction and fiber tracking}

\subsubsection{Fiber trajectory model}

If white matter fiber is composed of a train of vectors, as shown in Figure 1, each vector has one point $x\left(x \subset R^{3}\right)$ and one direction $v_{1: n}=\left\{v_{1} \ldots \ldots v_{n}\right\}, x_{1}$ is the seed voxel, then each point of the path can be defined as: 


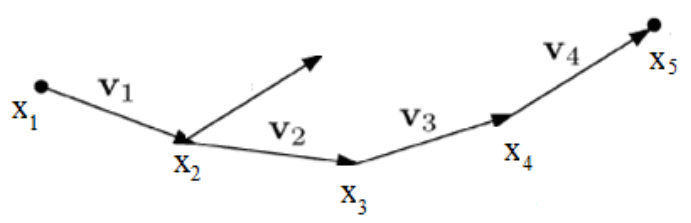

Fig. 1. A fiber path is composed of a train of vectors, and in the $x_{2}$ voxel, there are two directional peaks in the ODF. Therefore, the $x_{2}$ voxel has two possible directions in the path, but only the most probable one should be chosen.

$$
x_{k+1}=x_{k}+\lambda v_{k}
$$

where $\lambda$ represents a constant step size, $v_{k}$ represents the propagation direction in the voxel where $x_{k}$ is located, and the direction is defined by the ODF in the voxel. In QBI, one voxel ODF may have multiple directional peaks (such as $v_{2}$ in Figure 1), giving this voxel multiple choices for its next step. Eq. (1) indicates that each step only depends on previous step and on the current observation data, meaning that the fiber paths are Markov chains.

\subsubsection{Graph construction}

A graph $\mathrm{G}=[\mathrm{N}, \mathrm{E}]$ is defined by a set $\mathrm{N}$ of $\mathrm{n}$ elements called nodes, and a set $\mathrm{E}$ of e elements called edges. Edges link pairs of nodes. The number of elements of a set $\mathrm{N}$ is known as the cardinality of $\mathrm{N}$. Each edge $\mathrm{e} \in \mathrm{E}$ is assigned a number $\mathrm{w} \in \mathrm{R}$, and represents the weight of the edge, and the definition of the weight can be length, cost of transportation along the edge, time required to pass the edge, or the probability of its existence. An appropriate definition of weight can optimize the calculation of the shortest path between nodes, and in this paper can be interpreted as the brain white matter fiber bundles.

Suppose that $D$ represents the skull-stripped brain HARDI space, $D \subset R^{3}, \varepsilon$ denotes an arbitrary cubic voxel in $D$, and $\alpha$ denotes the center of the cubic voxel, which is also a node on the graph. Let $N$ denote the set of all nodes. For a given node, the edges are added to each vertex comprising a set of neighboring vertices. The neighboring vertices can be 26 or 98 vertices in the closest spatial proximity to a given node. Further, let $E$ denote the set of all edges. By combining all of the nodes and edges, we can construct a brain-weighted graph, as shown in Figure 2.

\subsubsection{Weights of graph edges}

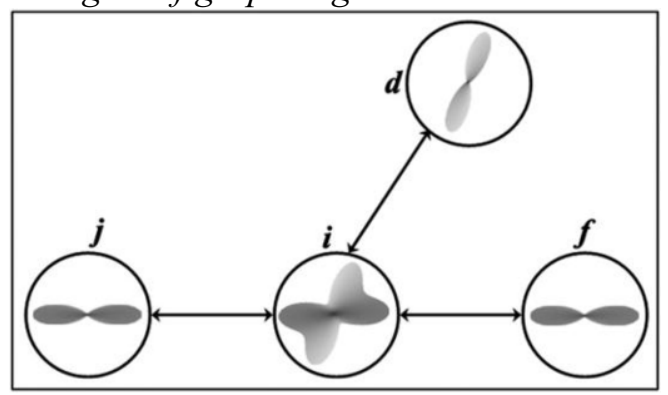

Fig. 2. Brain graph constructed in 3D-HARDI space, where $\mathrm{d}, \mathrm{f}, \mathrm{i}$ and $\mathrm{j}$ represent the nodes in the graph, and the arrows represents the edges in the graph. All Q-ball ODFs are sharpened. The fiber bundles in voxel i may cross, so the ODF has two peaks. The figure was provided by [17].

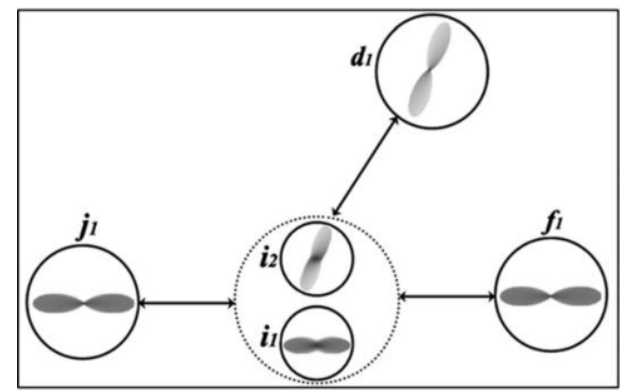

Fig. 3. The voxel with two ODF peaks is divided into two parts, and each part is considered to be a whole node in the graph and has a respective weight for their edges. The figure was provided by [17]. 
The most important issue is how to define the weights of the edges. This paper used the ODF and Bayesian model to calculate the weights of the edges between two nodes. If the ODF of the given voxel has multiple peaks, the corresponding node is divided into $k$ parts, and $k$ is equal to the number of ODF peaks. Each part is considered to be one normal node of the graph, as shown in Figure 3.

According to the number of the ODF peaks in each voxel, the weights of the edges have different computational equations. If a voxel's ODF has only one peak, then the weight will be calculated using the Bayesian model in two directions from current and previous nodes. If a voxel's ODF has multiple peaks, the ODF should be included in the following equation:

$$
w(i, j)=\left\{\begin{array}{lc}
\psi(i, j) & \text { ODF has one peak } \\
\psi(i, j) \bullet \theta\left(j, d_{k}\right) & \text { ODF has multiple peaks }
\end{array}\right.
$$

where $w(i, j)$ represents the weights of edges, i represents the previous voxel, $\mathrm{j}$ represents the current voxel, $\psi(i, j)$ represents the posterior distribution value between two voxels that is calculated using the Bayesian model, and $\theta\left(j, d_{k}\right)$ represents the ODF value of voxel $j$ in the $d_{k}$ orientation.

Brain tractography based on Bayesian models was first proposed by Friman, et al. [13]. They used a solid Bayesian theorem to model and estimate the probability functions. Our work is built of this model. We made several assumptions for the white matter fiber path:

(1) A fiber path has finite length and can be modeled as a train of vectors, as shown in Figure 1, where the path is represented by $v_{1: n}=\left\{\hat{v}_{1} \ldots . . \hat{v}_{n}\right\}$;

(2) All vectors have the same length;

(3) The vector $\hat{v}_{i}$ only depends on the previous vector $\hat{v}_{i-1}$, and is independent of the other vectors;

(4) $\Omega_{A}^{n}$ represents the set of all fiber paths that originate from a voxel or area $A$, whose length are $n$, and we can describe the probability of each fiber path via $p\left(v_{1: n}\right)$;

(5) The tensor model is used to describe the diffusion of weighted signals;

$$
\mu_{j}=\mu_{0} e^{-b_{j} \hat{g}_{j}^{T} D \hat{g}_{j}}
$$

(6) Prior knowledge about the parameters of the tensor model in the current voxel is independent of the previous direction;

(7) In the calculation of the posterior distribution function (pdf), the parameters are fixed to some values and expressed with dirac impulses.

While the vector $\hat{v}_{i-1}$ is determined, the posterior distribution function of current vector $\hat{v}_{i}$ can be modeled as:

$$
p\left(\hat{v}_{i}, \theta \mid \hat{v}_{i-1}, D\right)=\frac{p\left(D \mid \hat{v}_{i}, \theta\right) p\left(\hat{v}_{i} \mid \hat{v}_{i-1}\right) p(\theta)}{p(D)}
$$

where $D$ represents the diffusion measurement, and $\theta$ represents the set of parameters in tensor model. The $p(\mathrm{D})$ value normalizes the posterior distribution function and can be written as the integral of the 
numerator, so $p(D)$ is written:

$$
p(D)=\int_{\hat{v}_{i}, \theta} p\left(D \mid \hat{v}_{i}, \theta\right) p\left(\hat{v}_{i} \mid \hat{v}_{i-1}\right) p(\theta)
$$

By combining Eqs. (4) and (5), the posterior distribution function is written as:

$$
p\left(\hat{v}_{i}=\hat{v}_{k} \mid \hat{v}_{i-1}, D\right)=\frac{p\left(D \mid \hat{v}_{k}, \theta\right) p\left(\hat{v}_{k} \mid \hat{v}_{i-1}\right)}{\sum_{\hat{v}_{k} \in S} p\left(D \mid \hat{v}_{k}, \theta\right) p\left(\hat{v}_{k} \mid \hat{v}_{i-1}\right)}
$$

where $S$ represents the set of unit vectors distributed around the previous vector, and $\hat{v}_{k} \in S$. Joint distribution of data $D=\left[z_{1}, z_{2}, \ldots, z_{N}\right]$ is

$$
p(D \mid \hat{v}, \theta)=\prod_{j=1}^{N} \frac{\mu_{j}}{\sqrt{2 \pi \sigma^{2}}} e^{-\frac{\mu_{j}^{2}}{2 \sigma^{2}}\left(z_{j}-\ln \mu_{j}\right)^{2}}
$$

Given the previous vector $\hat{v}_{i-1}$, the probability function current vector $\hat{v}_{i}$ is

$$
p\left(\hat{v}_{i} \mid \hat{v}_{i-1}\right)=\left\{\begin{array}{lll}
\hat{v}_{i}^{T} \hat{v}_{i-1} & \text { while } & \hat{v}_{i}^{T} \hat{v}_{i-1} \geq 0 \\
0 & \text { while } & \hat{v}_{i}^{T} \hat{v}_{i-1}<0
\end{array}\right.
$$

The probability of the current vector decreases while the angle between the two vectors increases, and it reaches zero probability if the angle $>=90^{\circ}$.

Dirac impulses are the priors for parameters, so

$$
p(\theta)=\prod_{i=0}^{M} \delta\left(\theta_{i}-\hat{\theta}_{i}\right)
$$

Using the weights of the graph edges, the probability of each fiber path $p(S)$ can be calculated by:

$$
p(S)=\prod_{i=1}^{n} p\left(s_{1}\right) p\left(s_{2}\right) \ldots \ldots p\left(s_{n}\right)
$$

where the path $\mathrm{S}$ is composed of $n$ vectors, $s_{1}, s_{2}, \ldots \ldots, s_{n}$, and the probability of each vector is $p\left(s_{i}\right)$. Therefore, the fiber path from the given voxel to the destination voxel can be represented as the most probable path from these two voxels:

$$
p\left(S^{*}\right)=\underset{S \in \xi}{\arg \max } p(S)
$$


where $\xi$ represents all the paths from the given voxel to the destination voxel, and $\mathrm{S}^{*}$ is the path with the maximum probability.

\subsubsection{Fiber tracking based on graph theory}

Using graph theory, the brain white matter fiber path-tracking problem between two voxels can be transformed into the shortest path in a graph between two nodes. Building off the previous sections, the nodes, edges, and weights in graph are all constructed, and the shortest path between two nodes can be calculated using Dijkstra's algorithm.

Dijkstra's algorithm is as follows:

Suppose that in graph $G$, the nodes set are $N$, edges set $E$, weights set $W$, edge probabilities $p(e)$ for all $e \in E$, seed point $u$, target point $v, x_{e}$ denotes the point at which edge $e$ terminates, and $s_{u, v}^{*}$ denotes the shortest path. Then,

$\mathrm{d}(\mathrm{x})=-\infty$, for all $\mathrm{x} \in \mathrm{N} ; \quad \operatorname{prev}(\mathrm{x})=$ emtpy, for all $\mathrm{x} \in \mathrm{N} ; \quad \mathrm{d}(\mathrm{u})=0 ; \quad \mathrm{Q}=\mathrm{N}$;

while $\mathrm{Q}$ is not the empty set do

$\mathrm{Q}=\mathrm{Q} \backslash \mathrm{x}^{*}$, where $\mathrm{d}\left(\mathrm{x}^{*}\right)=\max _{\mathrm{x}} \in \mathrm{Q} \mathrm{d}(\mathrm{x})$;

for $\mathrm{e} \in \mathrm{N}_{\mathrm{x}^{*}}$ do

if $\mathrm{d}\left(\mathrm{x}^{*}\right)+\log \mathrm{p}(\mathrm{e})>\mathrm{d}\left(\mathrm{x}_{\mathrm{e}}\right)$ then

$$
\mathrm{d}\left(\mathrm{x}_{\mathrm{e}}\right)=\mathrm{d}\left(\mathrm{x}^{*}\right)+\log \mathrm{p}(\mathrm{e})
$$

end if $\operatorname{prev}\left(\mathrm{x}_{\mathrm{e}}\right)=\mathrm{x}^{*}$;

end for

end while

$\mathrm{x}=\mathrm{v}, \quad s_{u, v}^{*}=(\mathrm{v})$;

while $\mathrm{x} \neq \mathrm{u}$ do

$\mathrm{x}=\operatorname{prev}(\mathrm{x})$

Add $\mathrm{x}$ to the head of $s_{u, v}^{*}$

end while

\section{Experiments and results}

The experimental design of this study consisted of two parts: the phantom fiber tracking test and the real brain white matter fiber-tracking test. First, the method in this paper was used to reconstruct fiber tracking based on a phantom. Since the ground truth of the phantom's fiber tracking results was already known, the present method's accuracy could be clearly obtained via comparison. Then, the present method was used to reconstruct fiber tracking based on real brain white matter data in order to demonstrate a practical clinic result.

\subsection{Phantom fiber tracking test}

There are two main fibers in our phantom (Figure 4). One fiber is a straight tract that originates at point $\mathrm{A}$ and ends at point $\mathrm{B}$. The other fiber is a branching tract that originates at point $\mathrm{C}$ and branches into three points: D, E and F. These two fibers cross at point $G$, as shown in Figure 4. In the single-fiber voxel, DW signals are simulated based on the DTI model [20]. In the fiber crossing voxel, DW signals are simulated based on the mixture model [21]. No noise is added in the signals. 


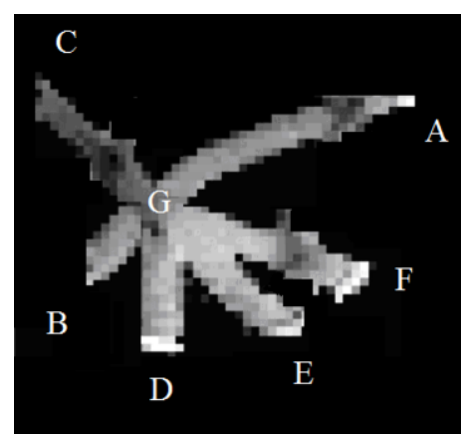

Fig. 4. Diagram of our phantom of white fiber bundles. One fiber is a straight tract that originates at point $\mathrm{A}$ and ends at point $\mathrm{B}$. The other fiber is a branching tract that originates at point $\mathrm{C}$ and branches into three points: $\mathrm{D}, \mathrm{E}$ and $\mathrm{F}$. These two fibers cross at point $\mathrm{G}$.

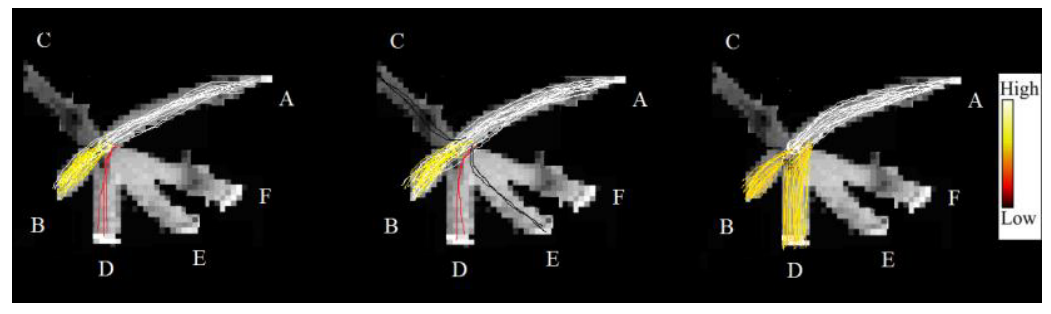

(a) our method

(b) GT

(c) FMT

Fig. 5. Comparison of the three tractography methods using phantom data. All the fiber paths originate from point $\mathrm{A}$ and are colour-coded with the path probabilities.

We then compared our method with the traditional graph tractography using DTI (GT) [14] and the fast marching tractography using QBI (FMT) [22]. These three methods are used to extract fiber paths in the phantom data. The paths are colour-coded by path probabilities (or connectivity in the case of FM).

First, point A was used as the seed point, and points B, C, D, E and F were used as target points. Since the seed point is in the straight tract, the fiber path along A-B should have higher probabilities than the other paths (A-C, A-D, A-E, and A-F). The tractography results of the three methods are shown in Figure 5. The GT method and our method extracted fiber paths A-B with much higher probabilities than it did for other fiber paths. Because these two methods both use graph theory to extract the fiber paths, they were able to accurately handle the fiber crossings. The FMT extracted fiber paths A-B and A-D with nearly identical probabilities, but due to the large crossing angle, almost no fiber bundles were extracted along the paths A-C, A-E and A-F.

Point $\mathrm{C}$ was then used as a seed point, and points $\mathrm{A}, \mathrm{B}, \mathrm{D}, \mathrm{E}$, and $\mathrm{F}$ were used as target points. Since the seed point was in the branching tract, the fiber paths along C-D, C-E and C-F should have almost the same probabilities, and almost zero probabilities for the other paths. The tractography results of the three methods are shown in Figure 6. The FMT and our method extracted fiber paths C-D, C-E and C-F with almost the same probabilities. Because these two methods both use QBI and ODF to characterize multiple fibers in a single voxel, they can accurately handle the problems associated with

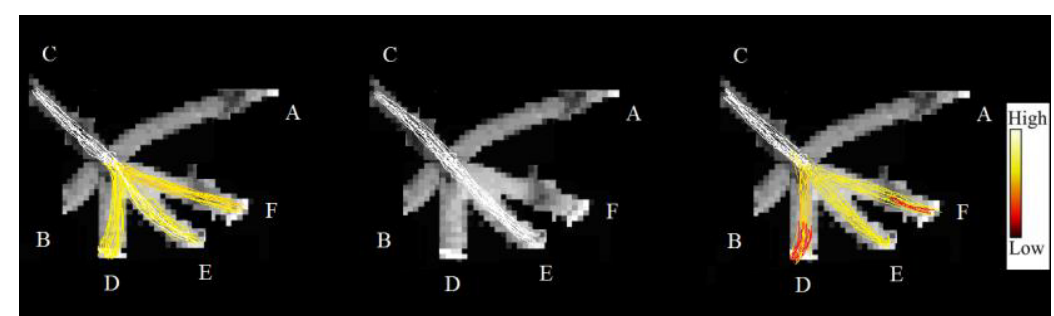

(a) our method (b) GT (c) FMT

Fig. 6. Comparison of the three tractography methods using phantom data. All fiber paths originate from point $\mathrm{C}$ and are colour-coded with the path probabilities. 


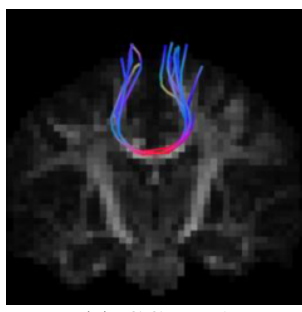

(a) CCtoM1

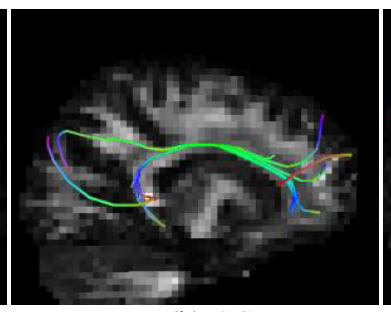

(b) CG

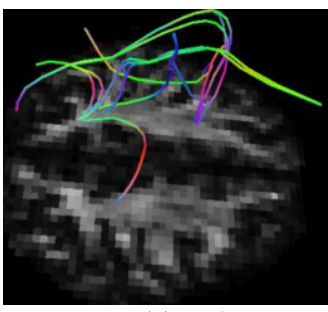

(c) IFO

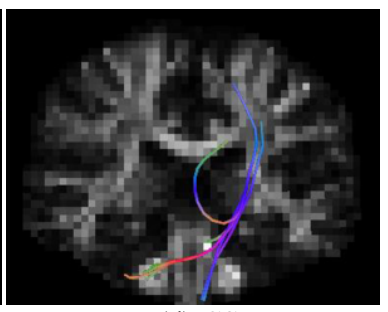

(d) CST

Fig. 7. Diagram of several well-known fiber bundles, (a) callosal fibers coming from the left motor cortex (CCtoM1), (b) the cingulum (CG), (c) the inferior fronto-occipital fasciculus (IFO), and (d) the corticospinal tracts to the left motor cortex (CST).
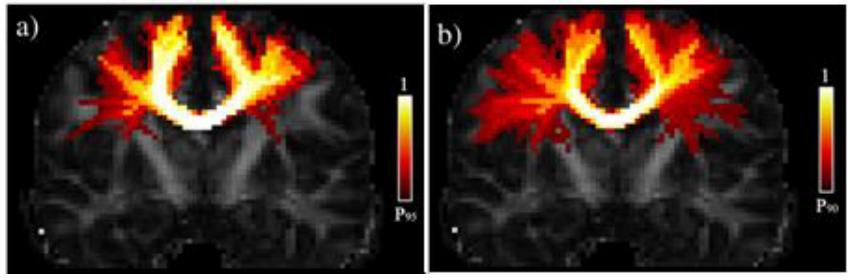

Fig. 8. Connection strength map of callosal fibers from Stamatios, et al. [17], (a) fiber tracking results using diffusion ODFs, (b) fiber tracking results using fiber orientation distribution functions (fODFs).

fiber branching. The GT method could only extract one fiber path, C-E, because the DT uses the tensor model whose ODF only has one peak in each voxel, and the GT could not handle the fiber branching.

Therefore, using both graph theory and QBI, our method was able to accurately handle the fiber crossing and branching.

\subsection{Real brain white matter fiber tracking test}

The real brain QBI data is from a healthy subject (male, 42 years-old). The local research ethics committee approved the testing, and informed consent was obtained. The QBI data was obtained from a Philips 3T Achieva clinical imaging system with 67 directions. A single-shot, spin-echo, echo-planar, diffusion-weighted sequence was used, and diffusion weighting data was obtained with $b=3000 \mathrm{~s} / \mathrm{mm}^{\mathrm{s}}$ ( $\mathrm{TE}=72 \mathrm{~ms}, \mathrm{TR}=15292 \mathrm{~ms}$ ). Slice thickness was $2 \mathrm{~mm}$.

Several regions of interest (ROI) were selected to extract well-known fiber bundles (Figure 7). These bundles are the callosal fibers coming from the left motor cortex (CCtoM1), the cingulum (CG), the inferior fronto-occipital fasciculus (IFO), and the corticospinal tracts to the left motor cortex (CST). Each of the bundles extracted by our method coincide with the anatomy and physiology of human brain structures. Therefore, the brain white matter fiber reconstruction result is able to provide important information and guidance for clinical and neuroscience research. Our fiber tracking results from the real DTI data were compared with data from Stamatios, et al. [17]. The callosal fibers tracking results from this study are shown in Figure 8. Following a comparison with our callosal fiber tracking results (Figure 7(a)), it is clear that the results of Stamatios, et al. still had several branch fibers that were not well handled.

\section{Conclusion}


This study presents a novel method for brain white matter tractography based on graph theory using QBI. Our method can accurately handle fiber crossing and branching with a singe voxel. The presented method can provide better results than other traditional methods when using phantom data, and provide important information and guidance for clinical and neuroscience research.

\section{Acknowledgment}

This paper was supported by National Natural Science Foundation of China (No.61101057) and the Fundamental Research Funds for the Central Universities (No. N130404027).

\section{References}

[1] P. Basser, J. Mattiello and D. LeBihan, MR diffusion tensor spectroscopy and imaging, Biophysical Journal 66 (1994), 259-267.

[2] B. Sebastiano, H.A.B. Miriam, K. Jan, et al., Segmentation of fiber tracts based on an accuracy analysis on diffusion tensor software phantoms, NeuroImage 55 (2011), 532-544.

[3] J. Pontabry, F. Rousseau and E. Oubel, Probabilistic tractography using Q-ball imaging andparticle filtering: Application to adult and in-utero fetal brain studies, Medical Image Analysis 17 (2013), 297-310.

[4] F. Ola, F. Gunnar and W. Carl-Fredrik, A Bayesian approach for stochastic white matter tractography, IEEE Transactions on Medical Imaging 25 (2006), 965-978.

[5] P.J. Basser, S. Pajevic and C. Pierpaoli, In vivo fiber tractography using DT-MRI data, Magnetic Resonance in Medicine 44 (2000), 625-632.

[6] P. Fillard, C. Poupn and J. Mangin, novel global tractography algorithm based on an adaptive spin glass model, Medical Image Computing and Computer-Assisted Intervention 5761 (2009), 927-934.

[7] S. Jbabdi, M. Woolrich, J. Andersson and T. Behrens, A bayesian framework for global tractography, NeuroImage 37 (2007), 116-129.

[8] S. Lifshits, A. Tamir and Y. Assaf, Combinatorial fiber-tracking of the human brain, NeuroImage 48 (2009), 532-540.

[9] P. Staempfli, T. Jaermann and G. Crelier, Resolving fiber crossing using advanced fast marching tractography based on diffusion tensor imaging, NeuroImage 30 (2006), 110-120.

[10] X. Wu, Q. Xu and L. Xu, Probabilistic white matter fiber tracking using particle filtering and von Mises-Fisher sampling, Medical Image Analysis 13 (2009), 5-18.

[11] T. Behrens, H.J. Berg and S. Jbabdi, Probabilistic diffusion tractography with multiple fibre orientations: What can we gain?, NeuroImage 34 (2007), 144-155.

[12] T. Behrens, M. Woolrich and M. Jenkinson, Characterization and propagation of uncertainty in diffusion-weighted MR imaging, Magnetic Resonance in Medicine 50 (2003), 1077-1088.

[13] O. Friman, G. Farneback and C. Westin, A bayesian approach for stochastic white matter tractography, IEEE Transactions on Medical Imaging 25 (2006), 965-978.

[14] Y. Iturria-Medina, E.J. Canales-Rodriguez and L. Melie Garcia, Characterizing brain anatomical connections using diffusion weighted MRI and graph theory, NeuroImage 36 (2007), 645-660.

[15] C. Frindel, M. Robini and J. Schaerer, A graph-based approach for automatic cardiac tractography, Magnetic Resonance in Medicine 64 (2010), 1215-1220.

[16] D.S. Tuch, Q-ball Imaging, Magnetic Resonance in Medicine 52 (2004), 1358-1372.

[17] N.S. Stamatios, B. Li and S.M. Pal, Brain tractography using Q-ball imaging and graph theory: Improved connectivities through fibre crossings via a model-based approach, NeuroImage 49 (2010), 2444-2456.

[18] M. Descoteaux, R. Deriche, T. Knosche and A. Anwander, Deterministic and probabilistic tractography based on complex fibre orientation distributions, IEEE Transactions on Medical Imaging 28 (2009), 269-286.

[19] I. Aganj, C. Lenglet and G. Sapiro, Odf maxima extraction in spherical harmonic representation via analytical search space reduction, Medical Image Computing and Computer-Assisted Intervention 6362 (2010), 84-91.

[20] P.J. Basser, J. Mattiello and D. Lebihan, Estimation of the effective self-diffusion tensor from the NMR spin echo, Journal of Magnetic Resonance 103 (1994), 247-254.

[21] P.A. Cook, M. Symms, P.A. Boulby and D.C. Alexander, Optimal acquisition orders of diffusion-weighted MRI 
measurements, Journal of Magnetic Resonance 25 (2007), 1051-1058.

[22] J.S. Campbell, K. Siddiqi and V.V. Rymar, Flow-based fiber tracking with diffusion tensor and q-ball data: Validation and comparison to principal diffusion direction techniques, NeuroImage 27 (2005), 725-736. 